\title{
Análisis de la relación entre variables morfométricas y biofísicas en la estimación de características probabilísticas para la oferta hídrica superficial en Colombia
}

\author{
Jaime Burbano-Girón",*, Efraín Domínguez², Oscar Barón-Ruiz ${ }^{3}$ \\ ${ }^{1}$ Facultad de Estudios Ambientales y Rurales, Pontificia Universidad Javeriana, Bogotá, Colombia \\ ${ }^{2}$ Departamento de Ecología y Territorio, Pontificia Universidad Javeriana, Bogotá, Colombia \\ ${ }^{3}$ Pregrado en Ecología, Pontificia Universidad Javeriana, Bogotá, Colombia
}

\begin{abstract}
Resumen
Actualmente, el recurso hídrico superficial enfrenta una alta presión antrópica por las transformaciones del paisaje natural y los altos niveles de demanda de agua de las actividades socioeconómicas. Ante esta situación, la modelación integrada del ciclo hidrológico se convierte en una herramienta necesaria para evaluar y predecir las consecuencias de las transformaciones del paisaje sobre los elementos del ciclo hidrológico. Su exitosa aplicación depende de la disponibilidad de información y la escala de estudio. Con base a lo anterior, se determinó que la información existente actualmente en el país no permite aplicar con rigurosidad ninguno de ellos, por ende se trabajó en un modelo estadístico de Análisis de Componentes Principales para relacionar información morfométrica, biofísica e hidrológica derivada para la caracterización de 421 cuencas generadas en Colombia. El 91\% de las cuencas tuvo un error relativo respecto al área de la cuenca reportada por el Instituto de Hidrología, Meteorología y Estudios ambientales (IDEAM) inferior al 15\%, y la significancia de las relaciones entre las variables hidrológicas, morfométricas y biofísicas fue en la gran mayoría de los casos significativa. Lo anterior valida aun las relaciones establecidas, señalando que los resultados del modelo estadístico construido, permiten estimar la oferta hídrica en lugares sin registro hidrológico a través de los parámetros morfométricos y biofísicos de las cuencas sin instrumentación hidrológica. (C) 2016. Acad. Colomb. Cienc. Ex. Fis. Nat.
\end{abstract}

Palabras clave: Modelamiento hidrológico integrado; Ciclo hidrológico y oferta hídrica superficial.

Analysis of the relationship between morphometric and biophysical variables in the estimation of probability characteristics for surface water supply in Colombia

\begin{abstract}
Currently, superficial hydrological resources face high anthropogenic pressures due to the natural landscape transformation and the high demand levels of water for socioeconomic activities. Given this situation, the integrated modeling of the hydrological cycle has become a necessary tool for evaluating and predicting the consequences of landscape transformation over elements of the water cycle. Its successful application depends on the available information and the scale of the study. Based on the above, it was determined that the existing information in the country doesn't allow the rigorous application of neither of them, therefore, efforts were made on a statistical model of Principal Component Analysis in order to relate derived morphometric, biophysical and hydrological information for the characterization of 421 basins generated in Colombia. As reported by the Hydrology, Meteorology and Environmental Studies Institute (IDEAM - acronym in Spanish) 91\% the basins had a relative error regarding the basin area lower than $15 \%$, the significance of the relationships between the morphometric, hydrological and biophysical variables was for most of the cases, significant. The above, validates even more the established relationships, highlighting that the results of the statistical model developed allows an estimation of the water demand in places without a hydrological register throughout morphometric and biophysical parameters of the basins without a hydrological instrumentation. (c) 2016. Acad. Colomb. Cienc. Ex. Fis. Nat.
\end{abstract}

Key words: Integrated hydrological modeling; Hydrological cycle and surface water supply.

\section{Introducción}

En el pasado se ha calificado a Colombia como una potencia en términos de su disponibilidad hídrica, sin embargo, debido a la creciente necesidad por el uso del espacio para su desarrollo socioeconómico se han desencadenado conflictos en términos de la protección de la oferta hídrica natural
(Domínguez, Rivera y Sarmiento, 2008). El crecimiento poblacional actual ha concentrado la demanda hídrica sobre regiones donde su oferta es escasa y en las cuales la presión

*Correspondencia:

Jaime Burbano-Girón, burbano.j@javeriana.edu.co

Recibido: 14 de octubre de 2015

Aceptado: 23 de agosto de 2016 
sobre el recurso hídrico ya registra altos requerimientos para mantener la población y la infraestructura (Etter y Van Wyngaarden, 2000; Etter y Villa, 2000; Domínguez, et al., 2008)

Ante esta situación y como apoyo a la gestión del recurso hídrico, la modelación integrada del ciclo hidrológico se convierte en una herramienta necesaria para evaluar y predecir las consecuencias de las transformaciones del paisaje sobre los elementos del ciclo hidrológico (Burbano-Girón, Domínguez y Etter, 2009). Las soluciones para el manejo del recurso hídrico hoy en día, dependen de la calidad de la información con que se toman las decisiones, derivada generalmente de datos y esfuerzos de modelamiento (Martin, LeBoeuf, Dobbins, Daniel y Abkowitz, 2005).

El modelamiento integrado del ciclo hidrológico tiene como unidad fundamental de estudio la cuenca, no obstante, los enfoques a este nivel se centran comúnmente en la simulación del proceso natural del flujo de agua que, aunque en algunas ocasiones puede modelarse junto a otros procesos como el transporte de sedimentos, químicos, nutrientes y organismos microbianos (Singh y Frevert, 2006a), deja de lado otros componentes del ciclo hidrológico. En la actualidad, existen muchos modelos matemáticos para el modelamiento hidrológico de cuencas con mayor o menor complejidad, dependiendo de las características del ciclo hidrológico que tienen cuenta; en este sentido son muy diversos y varían significativamente en términos de información y requerimientos computacionales (Singh, 1995; Singh y Frevert, 2002a; Singh y Frevert, 2002b).

La aplicación de modelos matemáticos a procesos hidrológicos se construye generalmente sobre enfoques deterministas, los cuales usan sistemas de ecuaciones diferenciales para representar las leyes físicas que describen el movimiento del agua; sin embargo, éstos también pueden ser obtenidos mediante la experiencia, o la estadística (Viessman y Lewis, 1996). De éstos pueden evidenciarse dos grandes deficiencias: la primera, la necesidad de un alto volumen de información requerida para un modelamiento integrado en cualquier cuenca en razón al número de variables, parámetros requeridos en el modelo y tamaño de la cuenca (escala), y la segunda, la baja disponibilidad de dicha información en países como Colombia en los que la dificultad de instrumentación y medición en la mayoría de cuencas es constante (Burbano-Girón, et al., 2009).

Otra aproximación al modelamiento hidrológico, son los modelos estocásticos. Éstos usan menor información en comparación a los deterministas, involucrando comúnmente componentes del ciclo hidrológico como: precipitación, evaporación y escorrentía, además de variables abióticas como la pendiente, y de parámetros físicos inherentes al sistema que describen características del proceso, obteniendo en todos los casos muy buenos ajustes en las probabilidades de escorrentía generadas para los sistemas estudiados (Frolov, Vyruchalkinay Solomonova, 2003; Frolov, 2006; Dolgonosov y Korchagin, 2007; Konovalov, 2007; Karasev, 2008).
Sin embargo, y pese a la diversidad de modelos encontrados, en el país actualmente no existe información completa que permita aplicar con rigurosidad ninguno de ellos. Algunos esfuerzos se han hecho en la aplicación de modelos determinísticos con grandes dificultades en la consecución y aplicación de la información (Burbano-Girón, et al., 2009), mientras en el caso estocástico la aplicación se ha llevado a cabo en cuencas donde por casos especiales la instrumentación lo ha permitido (Domínguez, 2004; Domínguez y Ivanona, 2005; Domínguez y Ramírez, 2010).

Ante las dificultades de información existentes, han surgido distintos enfoques para suplir los vacíos de información en la modelación hidrológica. Entre éstos, se destaca la construcción de modelos menos complejos basados en relaciones estadísticas, tales como los análisis de regresión múltiple, análisis factoriales de tipo componentes o coordenadas principales, o aproximaciones bayesianas (Lørup, Refsgaard y Mazvimavi 1998; Yang et al. 2007). La principal ventaja de éstos es que permiten integrar parámetros y variables para modelos tanto deterministas como estocásticos, reduciendo así los problemas de falta de información.

En función de esto, y tomando en cuenta que la oferta hídrica superficial constituye una noción probabilística e hidrológica (Domínguez, et al., 2008), en este trabajo se plantea entender la modelación integrada como aquella que puede considerar en forma concurrente las relaciones entre los elementos del balance hídrico, y los factores morfométricos y biofísicos de las cuencas, permitiendo construir indicadores de presión sobre el recurso hídrico, los cuales, en la mayoría de los casos, expresan en forma probabilista los elementos relacionados a la oferta hídrica.

En esta medida es clave resaltar que este trabajo apunta a presentar lineamientos iníciales para consolidar estrategias de modelamiento integrado adecuadas para las diferentes escalas de gestión del recurso hídrico, en especial para aquellas orientadas a la formulación de indicadores de presión sobre el recurso y para la evaluación de alteraciones del régimen hidrológico (Domínguez, et al., 2008).

Atendiendo a esta propuesta, se promueve la modelación estocástica del ciclo hidrológico cómo una herramienta adecuada para el fin propuesto. Cómo un primer adelanto, este trabajo analiza las relaciones estadísticas posibles entre los parámetros morfométricos y biofísicos de las cuencas y las características probabilísticas de la oferta hídrica superficial en Colombia hidrológico (Domínguez, et al., 2008).

Este estudio recopila información morfométrica y biofísica necesaria para la caracterización de 421 cuencas de Colombia (Figura 1), asociadas a las 421 estaciones involucradas en el Estudio Nacional del Agua 2008 (Domínguez, et al., 2008), con el fin de analizar las relaciones estadísticas entre los parámetros morfométricos y biofísicos de las cuencas y las características probabilísticas de la oferta hídrica superficial en Colombia. 


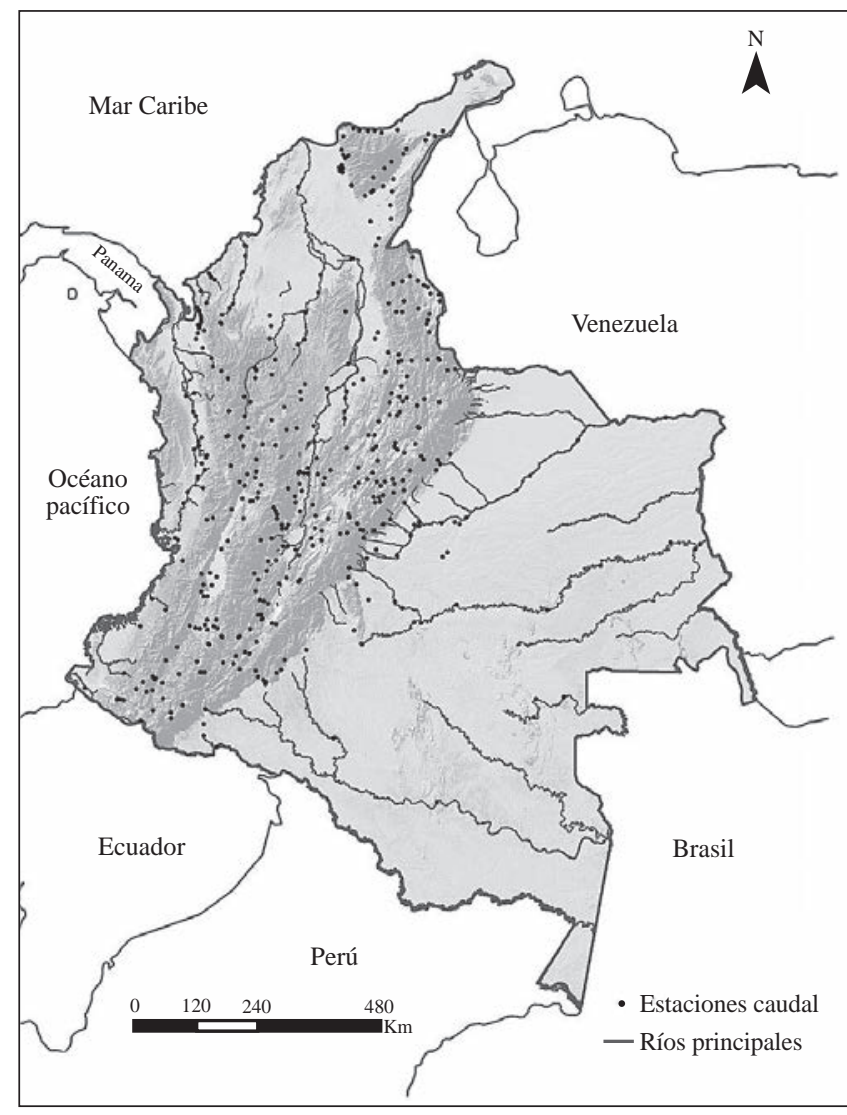

Figura 1. Área de estudio y estaciones hidrológicas usadas.

\section{Materiales y Métodos}

\section{Área de Estudio}

El área de estudio corresponde a las cuencas generadas para 421 estaciones de mediciones de caudal, involucradas en el Estudio Nacional del Agua (Colombia) (Domínguez, et al., 2008; IDEAM, 2008; Domínguez, Moreno y Ivanona, 2010) (Figura 1).

Colombia abarca aproximadamente 1142000 km² de territorio continental distribuidos entre los 0 y 5800 msnm, con precipitaciones que aproximadamente varían entre 250 y 11300 mm promedio anual, y temperaturas medias promedio anuales entre 0 y $30^{\circ} \mathrm{C}$ aproximadamente (IGAC, 2002). Climáticamente Colombia es muy compleja esencialmente por su ubicación geográfica y topografía. Para la mayoría de regiones del país se pueden distinguir dos periodos secos con predominancia de altas temperaturas y dos periodos húmedos con abundante pluviosidad, los cuales son constantemente influenciados por los efectos de El Niño y La Niña, y muy probablemente en la actualidad el cambio climático (Mesa, Poveda y Carvajal, 1997).

Esta variabilidad en las condiciones climáticas y biofísicas del país, se refleja en la diversidad hidrológica y morfométrica de sus cuencas. En esta medida, las áreas aferentes asociadas a las estaciones del estudio nacional del agua muestran una amplia diversidad en términos de tamaño, altura, pendiente, y valores de caudal y escorrentía, lo que abastece el soporte hidrométrico de cada uno de las variables de este estudio.

\section{Delimitación de Cuencas}

El proceso de delimitación de cuecas consistió en 4 etapas: a) La elección del Modelo de Elevación Digital (DEM por sus siglas en inglés) a usar. b) La reubicación de las estaciones, ya que no coincidían con los ríos generados por ninguno de los DEMs usados. c) La delimitación de las cuencas. d) Y la nueva reubicación de los puntos de las estaciones hasta encontrar un error relativo inferior al $15 \%$ en el área de la cuenca reportada por el Catálogo de la Red Hidrometeorológica y Ambiental Nacional de Estaciones del IDEAM.

a) Elección del Modelo de Elevación Digital

Paso previo a la delimitación de todas las cuencas se hicieron delimitaciones para distintas cuencas seleccionadas al azar probando 3 DEMs diferentes: SRTM 90m, SRTM 90m corregido (Jarvis et al, 2004) y Aster-GDEM (METI/ ERSDAC, USGS/EROS 2009) 90m (Originalmente de $30 \mathrm{~m}$ remuestreado a $90 \mathrm{~m}$ ). En esta exploración se determinó que los mejores ajustes entre la red hídrica cartográfica y la red hídrica generada por el DEM, así como del área para la cuenca reportada por el IDEAM, fueron los obtenidos por el DEM SRTM 90m, seguidos por el Aster-GDEM, por lo que se decidió trabajar con el primero. Sin embargo, se usó el segundo en la delimitación de algunas cuencas en las que el DEM SRTM 90m no tenía cobertura, debido a que estaba delimitado exclusivamente al área colombiana.

b) Reubicación de Estaciones

Las estaciones tuvieron que desplazarse de su ubicación inicial suministrada por las coordenadas del Catálogo de la Red Hidrometeorológica y Ambiental Nacional de Estaciones del IDEAM, con el fin de ajustarse a la red hídrica generada por el DEM a usarse (Figura 2).

c) Delimitación de Cuencas

La delimitación de cuencas se realizó utilizando HECGeoHMS 4.2 (USACE-HEC 2009) como extensión para ArcGIS 9.3 (ESRI 2008), con base a 2 Modelos de Elevación Digital: SRTM 90m y Aster-GDEM 90m (Originalmente de $30 \mathrm{~m}$ remuestreado a $90 \mathrm{~m}$ ). El 98\% de las cuencas (413 de 421) se delimitaron usado el producto SRTM, mientras en el $2 \%$ restante se decidió usar el producto Aster-GDEM con base a la disponibilidad de información fuera del territorio colombiano, con el fin de que la cuenca tuviera un límite de acuerdo a su morfometría real, ya que el producto SRTM estaba delimitado exclusivamente al área del país.

d) Nueva Reubicación de Estaciones

Se repitieron los pasos b) y c) hasta encontrar un error relativo inferior al $15 \%$ en el área de la cuenca reportada por el Catálogo de la Red Hidrometeorológica y Ambiental Nacional de Estaciones del IDEAM.

\section{Definición del Modelo}

Se eligió un modelo estadístico de Análisis de Componentes Principales (ACP) para integrar la información de las distintas variables de tipo morfométrico y biofísico. El ACP 


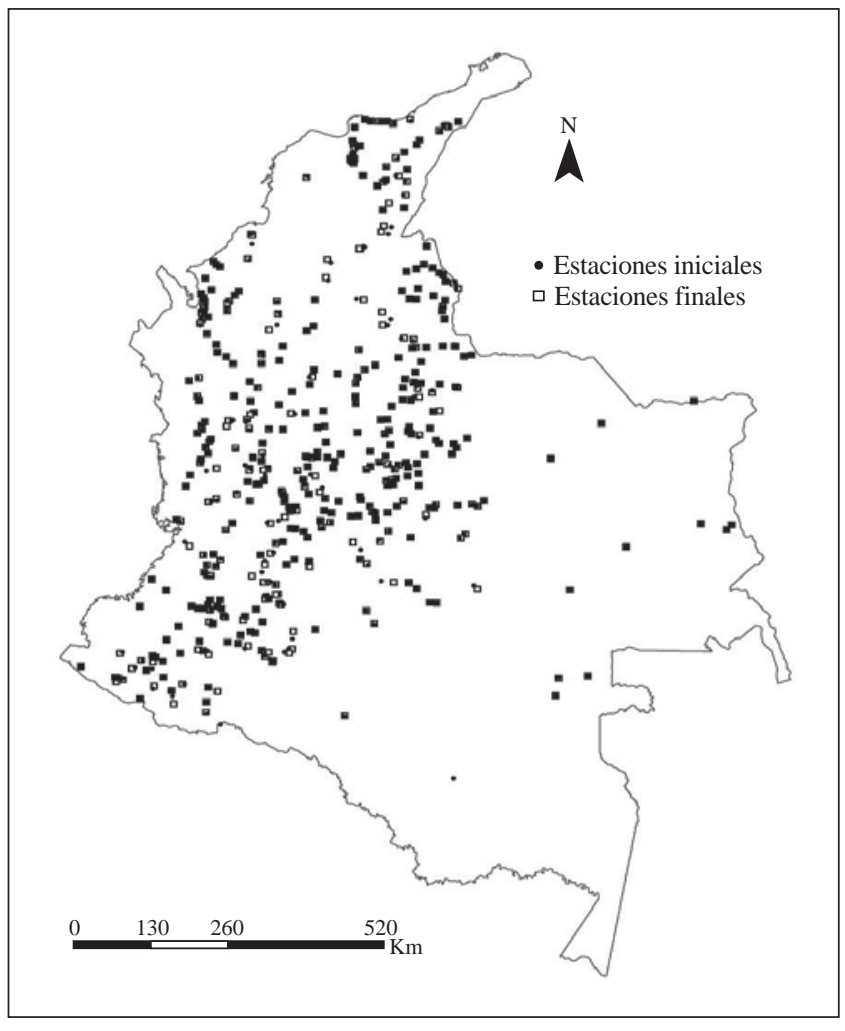

Figura 2. Movimiento de Estaciones en el Área de Estudio

como técnica de síntesis de información reduce la dimensión, es decir, el número de variables, por lo que, ante una gran cantidad de variables, el objetivo será resumirlas a un menor número perdiendo la menor cantidad de información posible. Adicionalmente mediante esta técnica se logran ver las relaciones que se establecen entre las variables, siendo muy útil si se pretende explicar relaciones de causalidad e interdependencia entre las mismas (Peña, 2002).

\section{Morfometría de las Cuencas}

Cada y río y su cuenca pueden ser caracterizados con indicadores cuantitativos de sus características morfométricas, para este trabajo se usaron 21 índices morfométricos de acuerdo a la propuesta de Samokhin y Saloviov (1980). Todas éstas fueron calculadas mediante operaciones de análisis espacial usando ArcGIS 9.3 (ESRI, 2008), y programación en lenguaje R (R Development Core Team, 2011) y Python (Python Software Foundation, 2008). A continuación, se detallan las 17 métricas morfométricas calculadas.

La (1) longitud del cauce principal $\boldsymbol{L}_{\boldsymbol{t}}$, es la distancia del recorrido que hace el cauce principal desde su nacimiento hasta su desembocadura medida en kilómetros. Usualmente $\boldsymbol{L}_{\boldsymbol{t}}$ presenta un grado de sinuosidad, el cual se representa a través del (2) coeficiente de sinuosidad $\boldsymbol{K}_{\boldsymbol{s}}$ que constituye la relación entre la distancia total configurada por el recorrido de la corriente $\boldsymbol{L}_{\boldsymbol{t}}$, sobre la (3) distancia lineal $\boldsymbol{L}_{\boldsymbol{l}}$ desde el nacimiento hasta la desembocadura: $\boldsymbol{K}_{\boldsymbol{s}}=\boldsymbol{L}_{\boldsymbol{t}} / \boldsymbol{L}_{\mathbf{l}}$
El (4) área aferente $\boldsymbol{A}$ de la cuenca, es el territorio sobre el que yace el cauce principal y del cual recolecta sus aguas, y se mide en kilómetros cuadrados. La (5) densidad de la red hidrográfica $\boldsymbol{D}$ expresa la longitud de red correspondiente a un área unitaria en kilómetros cuadrados. Se define como: $D=\sum_{i=1}^{n} L_{t_{i}} / A$, donde: $n$ es el número de corrientes visibles en la cartografía disponible. Con base en este parámetro se puede definir una aproximación de la (6) longitud media de las laderas en la cuenca. $L_{\mathbf{l a d}}=\mathbf{1 / 2 D}$

El (7) ancho medio de la cuenca $\boldsymbol{B}$, se determina como el cociente entre el área de la cuenca y la longitud lineal de la corriente principal: $B=A / L_{t}$. La (8) elongación de la cuenca o coeficiente de forma $\sigma$ se calcula como el cociente entre la longitud lineal de la corriente principal $\boldsymbol{L}_{\boldsymbol{l}}$ y el ancho medio de la cuenca $B: \sigma=L_{t} / B$. El (9) coeficiente de compacidad $k_{C}$ es la relación entre la longitud del perímetro de la cuenca $p_{\text {cuenca }} \mathrm{y}$ el perímetro de un círculo con área equivalente a la de la cuenca: $k_{c}: \sigma=0.28$ cuenca $\left(p_{\text {cuenca }} / B \sqrt{ } A\right)$.

La (10) altura media del cauce principal $H_{\text {cauce, }}$ se calcula como la altura promedio de cada tramo del cauce principal. La (11) pendiente media del cauce principal $I_{\text {cauce }}$ es el promedio de los cocientes entre los incrementos de alturas y los incrementos de longitud de la corriente para un tramo determinado. La (12) Altura media de la cuenca $H_{\text {cuenca }}$ y la (13) pendiente media de la cuenca $I_{\text {cauce }}$ corresponden a al promedio de las alturas y las pendientes dentro del área aferente de la cuenca $A$.

La (14) profundidad de disección de la cuenca $H_{\text {dis }}$, representa la diferencia entre la altura media de la cuenca $H_{\text {cuenca }}$ y la altura media del perfil longitudinal del río principal $H_{\text {cauce }}: H_{\text {dis }}=H_{\text {cuenca }}-H_{\text {cauce }}$. El (15) nivel de disección del relieve $N_{\text {dis }}$ resulta del doble producto de la densidad hidrográfica por la profundidad de disección: $N_{\text {dis }}=2 \mathrm{DH}_{\text {dis }}$.

El (16) número de orden de la cuenca $N$ se determina con base en el número de orden máximo obtenido para el cauce principal de la red hídrica de la cuenca. El orden de los ríos se calcula con base en el método propuesto por Strahler en 1952, donde los ríos en su nacimiento tienen valor de orden 1 y estos aumentan de orden solo cuando 2 o más ríos de un mismo orden se cruzan. Por lo tanto, la intersección de un río de primer orden y un río de segundo orden mantendrá el río de segundo orden, mientras si se unen 2 o más ríos de segundo orden el río resultante será de tercer orden. El (17) radio o coeficiente de Bifurcación $\boldsymbol{R} \boldsymbol{b}$ es el cociente entre el número de cauces $\boldsymbol{N}$ de orden $\boldsymbol{i}$ y el número de cauces $\boldsymbol{N}$ de orden inmediatamente superior $i+1: R b_{i, i+1}=N_{i} / N_{i+1}$.

\section{Variables Biofísicas}

Se incluyeron variables biofísicas con las que se pudiera inferir directrices en términos de conservación del recurso hídrico. Todas las variables usadas (Tabla 1) son producto de análisis espaciales en ArcGIS 9.3 (ESRI, 2008) con variables espacializadas para Colombia tomadas del Instituto Geográfico Agustín Codazzi (IGAC). 


\section{Variables Hidrológicas}

Se calcularon las siguientes variables hidrológicas relacionadas a la oferta hídrica: caudal medio anual (QmedA) y diario (QmedD), el coeficiente de variación anual (CvA) y diario (CvD), el coeficiente de asimetría anual (CsA) y diario (CsD), la probabilidad de excedencia del $97.5 \%$ para el caudal medio anual (Qa97.5), y mínimo diario (Qm97.5), y la escorrentía media anual (Ea97.5) y mínima diaria (Em97.5) (Domínguez, et al. 2009), el caudal modal anual (QmdA) y el

Tabla 1. Variables biofísicas calculadas.

\begin{tabular}{ll} 
Variable & Descripción \\
\hline $\begin{array}{l}\text { Proporción de } \\
\begin{array}{l}\text { Cobertura Boscosa } \\
\text { en la Cuenca }\end{array}\end{array}$ & $\begin{array}{l}\text { Proporción entre el área de cobertura boscosa (Mapa } \\
\text { de Ecosistemas de Colombia) en la cuenca y su área } \\
\text { total. }\end{array}$ \\
$\begin{array}{l}\text { Proporción de } \\
\begin{array}{l}\text { Espejos de Agua } \\
\text { en la Cuenca }\end{array}\end{array}$ & $\begin{array}{l}\text { Proporción entre el área de cuerpos de agua, } \\
\text { ciénagas y represas en la cuenca y su área total. }\end{array}$ \\
$\begin{array}{l}\text { Proporción de } \\
\text { Centros Poblados } \\
\text { en la Cuenca }\end{array}$ & $\begin{array}{l}\text { Proporción entre el área de cobertura de centros } \\
\text { poblados en la cuenca y su área total en la cuenca } \\
\text { y su área total. }\end{array}$
\end{tabular}

caudal modal diario de estiaje (QmdED), la escorrentía anual media (Emed) y modal (Emd), el coeficiente de regulación $(\mathrm{Kr})$ que representa la relación del área contenida entre el hidrograma de diarios y su línea de valor medio, y el número de rezagos hasta que se hace no significativa la correlación en los valores de caudal diario (M).

\section{Resultados}

\section{Delimitación de Cuencas}

En la Figura 3 se muestran las cuencas generadas y sus respectivos ríos principales para las 421 estaciones involucradas en el Estudio Nacional del Agua (Colombia) (IDEAM, 2008). Para el 97\% (407 de 421) de las cuencas se encontró reporte del valor de su área en el Catálogo de la Red Hidrometeorológica y Ambiental Nacional de Estaciones del IDEAM, por lo que se pudo evaluar el ajuste de las cuencas delimitadas con base al error relativo obtenido (Figura 4). En general se encontraron muy buenos ajustes. El $64 \%$ (260 de 407) de las cuencas tuvo un error relativo absoluto inferior al 6\%, el 81\% (330 de 407) inferior al 10\%, y el $91 \%$ inferior al $15 \%$. El 9\% restantes (4 de 407 ) no pudieron ser ajustadas a un error menor al $15 \%$.

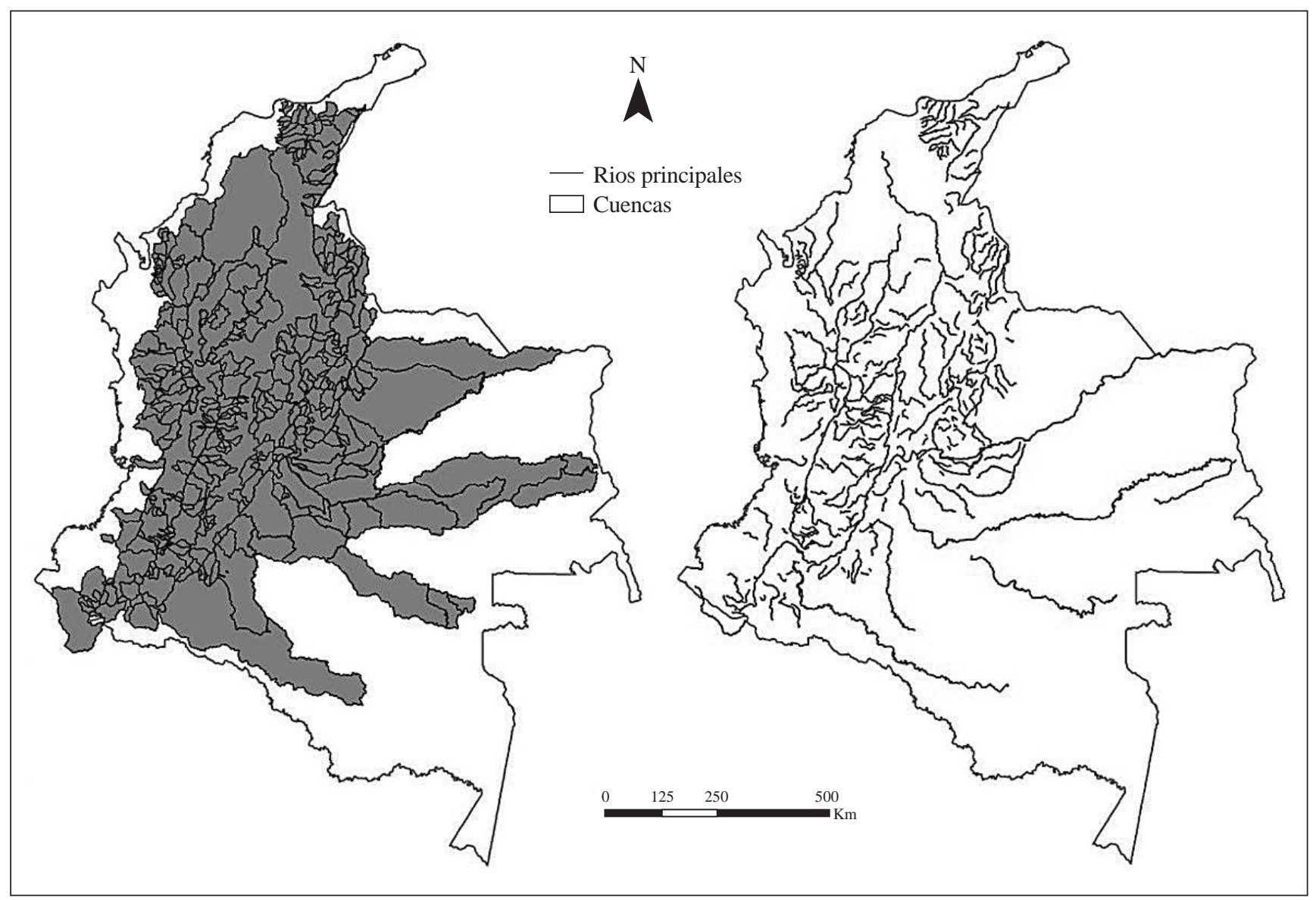

Figura 3. Cuencas y Cauces Principales generados para las 421 estaciones de estudio. Los cauces principales aparentan estar incompletos porque son los correspondientes al área aferente de la cuenca, la cual no necesariamente se cierra en la desombacadura de los ríos, sino donde se encuentra la estación correspondiente. 


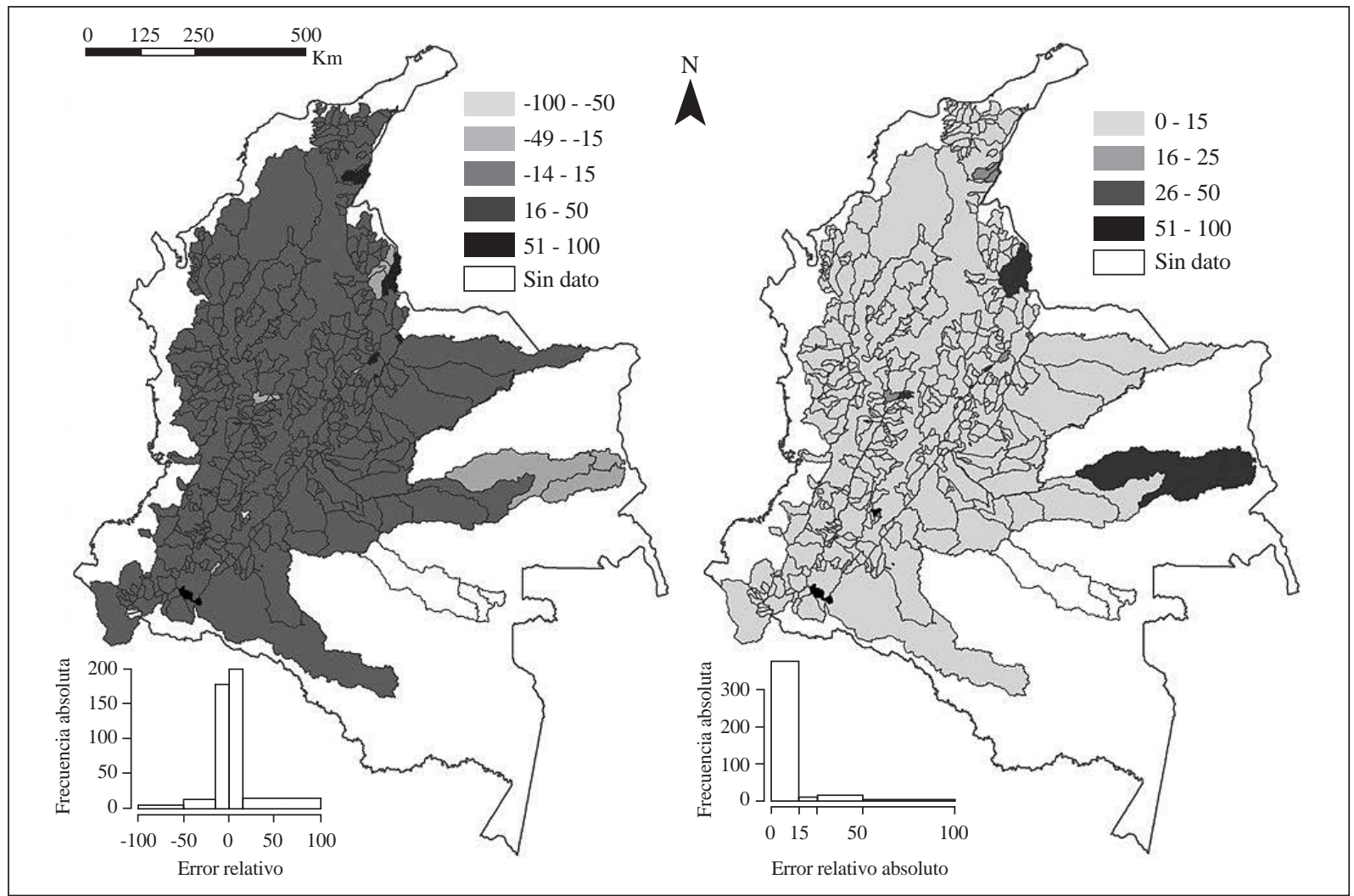

Figura 4. Error relativo (izquierda) y error relativo absoluto (derecha) en porcentaje para el ajuste del área en cada cuenca.

\section{Variables Morfométricas y Biofisicas}

En la Figura 5 se muestra la morfometría para la mayoría (solo las visibles) de las 421 cuencas. Es evidenciable que existen parámetros muy similares en su patrón espacial, por ejemplo: la densidad de la red hídrica, el coeficiente de forma y la longitud media de las laderas, el ancho de la cuenca y el coeficiente de compacidad, y la profundidad de disección y el nivel de disección. Por el contrario, las variables biofísicas muestran comportamientos diferentes entre ellas y en relación a los parámetros morfométricos. Lo anterior indica que existe una relación directa entre muchos aspectos de la morfometría, pero a su vez éstas son inversas con las variables biofísicas.

\section{Modelo Estadístico}

Se encontraron relaciones muy fuertes, confirmando lo encontrado en la descripción de las variables morfométricas y biofísicas (Tabla 1S, http://www.raccefyn.co/index. $\mathrm{php} / \mathrm{raccefyn} / \mathrm{article} /$ downloadSuppFile/291/1666). Por ejemplo, se encuentran muy relacionados la densidad de la red hídrica, el coeficiente de forma y la longitud media de las laderas, el ancho de la cuenca y el coeficiente de compacidad, la longitud del cauce, el área y el perímetro, la profundidad de disección y el nivel de disección, y los distintos valores de caudal entre ellos y con el área de la cuenca, mientras las variables biofísicas no muestran una relación clara.
En la Tabla 2 se muestran la desviación estándar de cada componente (valores propios) y su varianza explicada encontrada en el ACP. De ésta se puede inferir que el porcentaje de varianza explicado óptimo donde los valores propios para cada componente son mayor a 1 (Peña, 2002), se encuentran hasta el componente 10, explicando aproximadamente el $83 \%$ de la varianza en las relaciones presentadas para los valores de caudal y las variables morfométricas y biofísicas.

En la Figura 6 se muestran las relaciones presentadas en cada uno de los 10 componentes tomando como base en el eje X el Componente 1. Es recurrente la cercana correlación entre las variables relacionadas con el caudal, el área de la cuenca y longitud del río, siendo también muy cercanos a éstas el orden del río, los coeficientes de sinuosidad y compacidad, y el ancho medio de la cuenca.

El componente uno separa grupos de parámetros relacionados a la magnitud de la oferta en años de humedad media y baja (Qa97.5, Qm97.5, QmdA, QmdED, Emin97.5, Ea97.5), y su variabilidad (CvA, CvD, CsA, CsD). Con la magnitud de la oferta se asocian el ancho medio de la cuenca (B), el Número de Horton, el radio de autocorrelación diaria (M), El Area, la sinuosidad del cauce principal, la profundidad de disección de la cuenca (Hdis), la longitud del cauce principal (lt) y la proporción de bosque (Bos). Mientras que con la variabilidad se asocian características de la rugosidad del relieve cómo las pendientes y las alturas medias del cauce principal y la cuenca (lCue, lcau, Hcue, Hcau). 

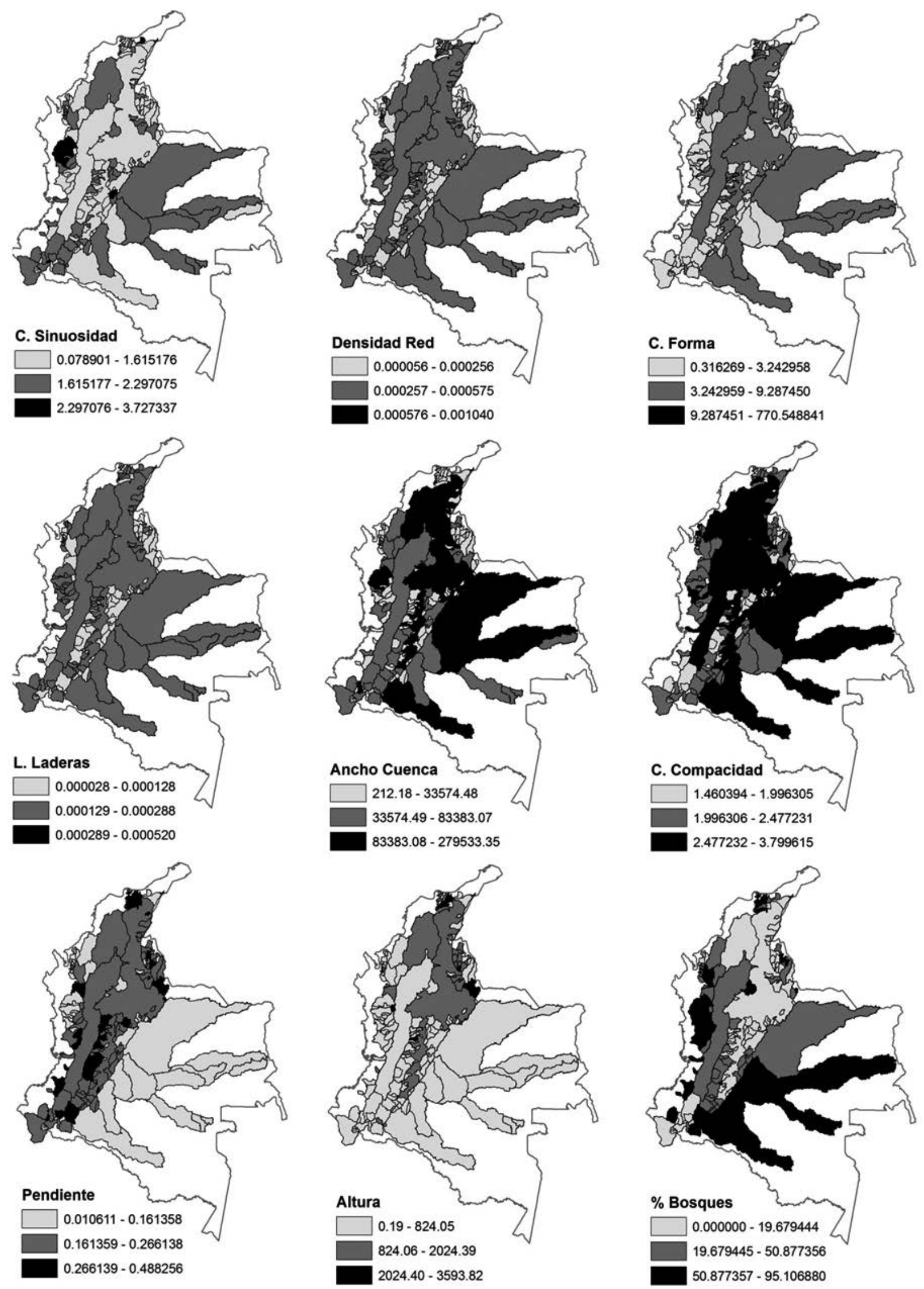

Figura 5. Valores de las variables morfometricas y biofísicas calculadas para las 421 cuencas. 
El componente dos, en lo relacionado a la oferta hídrica superficial, separa las características de magnitud (Volumen y caudal) de las de rendimiento (Escorrentía superficial). A la magnitud de la oferta se le asocian las características morfométricas relacionadas a geometría y tamaño, mientras que a las de rendimiento sólo se le asocia la proporción de Bosque. A su vez, los componentes dos y tres disocian los parámetros que influyen sobre la variabilidad de la oferta, ente ellos la pendiente media de la cuenca y el cauce principal (1Cue, ICau) y las alturas medias de la cuenca y el cauce principal (HCue, HCau). Estos componentes expresan el nivel de rugosidad de la topografía.

El componente 4 expresa la relación inversamente proporcional entre características de rugosidad del relieve (lCue, lcau, Hcue, Hcau) y las características geométricas de los cauces principales (N, Kc y Ks). El componente 5 refleja la relación entre profundidad de disección y Altura media de la cuenca. Los componentes restantes reflejan características particulares de las variables a las que se asocia un poder de explicación específico menor al 5\%.

Tabla 2. Valores propios y su varianza explicada obtenidos en el ACP.

\begin{tabular}{|c|c|c|c|}
\hline Componente & Desv.St & $\begin{array}{c}\% \text { de } \\
\text { Varianza Explicada }\end{array}$ & $\begin{array}{c}\text { \% de Varianza } \\
\text { Explicada Acumulada }\end{array}$ \\
\hline 1 & 3.22 & 32.47 & 32.47 \\
\hline 2 & 1.99 & 12.35 & 44.82 \\
\hline 3 & 1.57 & 7.67 & 52.49 \\
\hline 4 & 1.47 & 6.79 & 59.28 \\
\hline 5 & 1.30 & 5.25 & 64.53 \\
\hline 6 & 1.20 & 4.48 & 69.01 \\
\hline 7 & 1.16 & 4.18 & 73.18 \\
\hline 8 & 1.06 & 3.49 & 76.67 \\
\hline 9 & 1.04 & 3.40 & 80.07 \\
\hline 10 & 1.01 & 3.21 & 83.28 \\
\hline 11 & 0.89 & 2.50 & 85.79 \\
\hline 12 & 0.85 & 2.25 & 88.03 \\
\hline 13 & 0.82 & 2.08 & 90.12 \\
\hline 14 & 0.71 & 1.57 & 91.69 \\
\hline 15 & 0.67 & 1.39 & 93.08 \\
\hline 16 & 0.66 & 1.37 & 94.45 \\
\hline 17 & 0.59 & 1.10 & 95.55 \\
\hline 18 & 0.58 & 1.03 & 96.58 \\
\hline 19 & 0.54 & 0.91 & 97.49 \\
\hline 20 & 0.44 & 0.61 & 98.10 \\
\hline 21 & 0.41 & 0.54 & 98.64 \\
\hline 22 & 0.36 & 0.40 & 99.04 \\
\hline 23 & 0.33 & 0.34 & 99.38 \\
\hline 24 & 0.29 & 0.27 & 99.65 \\
\hline 25 & 0.24 & 0.19 & 99.84 \\
\hline 26 & 0.15 & 0.07 & 99.91 \\
\hline 27 & 0.12 & 0.05 & 99.96 \\
\hline 28 & 0.10 & 0.03 & 99.99 \\
\hline 29 & 0.05 & 0.01 & 100.00 \\
\hline 30 & 0.02 & 0.00 & 100.00 \\
\hline 31 & 0.01 & 0.00 & 100.00 \\
\hline 32 & 0.00 & 0.00 & 100.00 \\
\hline
\end{tabular}

En general, se puede inferir una asociación fuerte entre la altura y pendiente del cauce y la cuenca, la cual es inversa a los valores de caudal y área, es decir, a mayores valores de área y caudal deben esperarse valores bajos en la pendiente y altura media, tanto para el cauce como la cuenca.

Parece existir una aparente relación inversa entre los coeficientes de variación y asimetría (que a su vez están muy asociados) con las variables relacionadas al caudal, con lo que aparentemente se podría pensar en que cuencas de mayor tamaño donde los valores de caudal son mayores, tienden a ser más estables, lo cual es clave a la hora de evaluar pronósticos.

Las variables biofísicas: proporción de cuerpos de agua y centros poblados, no parecen tener una relación clara con ninguna variable hidrológica o morfómétrica, mientras la proporción de bosque muestra una relación de tipo directo con la escorrentía lo que vislumbra implicaciones en términos de conservación del recurso hídrico, puesto que a mayores valores de escorrentía encontrados se pueden ver mayores proporciones de bosque en las cuencas.

Las relaciones más fuertes dadas por el ACP se presentan en relación al valor del caudal y el tamaño de la cuenca. Como era de esperarse cuencas de mayor tamaño acumulan más agua debido a que acumulan más ríos de orden superior y por ende de mayor longitud. Así mismo las cuencas presentarán mayor sinuosidad debido a que contienen relieves más disectados por las variaciones desde las zonas altas a las a bajas.

La separación que muestra el ACP entre características más ligadas a la hidrología de la cuenca como el área, el orden del río, los coeficientes de sinuosidad y compacidad, y el ancho medio de la cuenca, respecto de las que son más ligadas a los parámetros del relieve como la altura y la pendiente, deja ver en algunos casos una relación muy lógica donde aparentemente las zonas en donde el caudal es mayor son aquellas donde las pendientes y alturas son menores, característica propia de las cuencas bajas donde ocurren las desembocaduras a los ríos de orden mayor y donde por su puesto se acumula igualmente un caudal mayor.

Adicionalmente éstas últimas características relacionadas al paisaje son muy asociadas entre sí, indicando que cualquiera de éstas, pendiente o altura, del cauce o de la cuenca, pueden ser buenos descriptores de su relieve. Las variables de profundidad de disección de la cuenca y cuerpos de agua también se encuentran muy relacionadas, junto con la densidad de la red hidrográfica y el ancho de la cuenca indicando una forma característica de la cuenca.

\section{Discusión}

Los resultados obtenidos señalan que los modelos estadísticos construidos permiten estimar la oferta hídrica en lugares sin registro hidrológico a través de los parámetros morfométricos y biofísicos de las cuencas sin instrumentación hidrológica. Éstos son respaldados en primera medida por los buenos ajustes obtenidos en la delimitación de las cuencas, lo que permite pensar que las características 

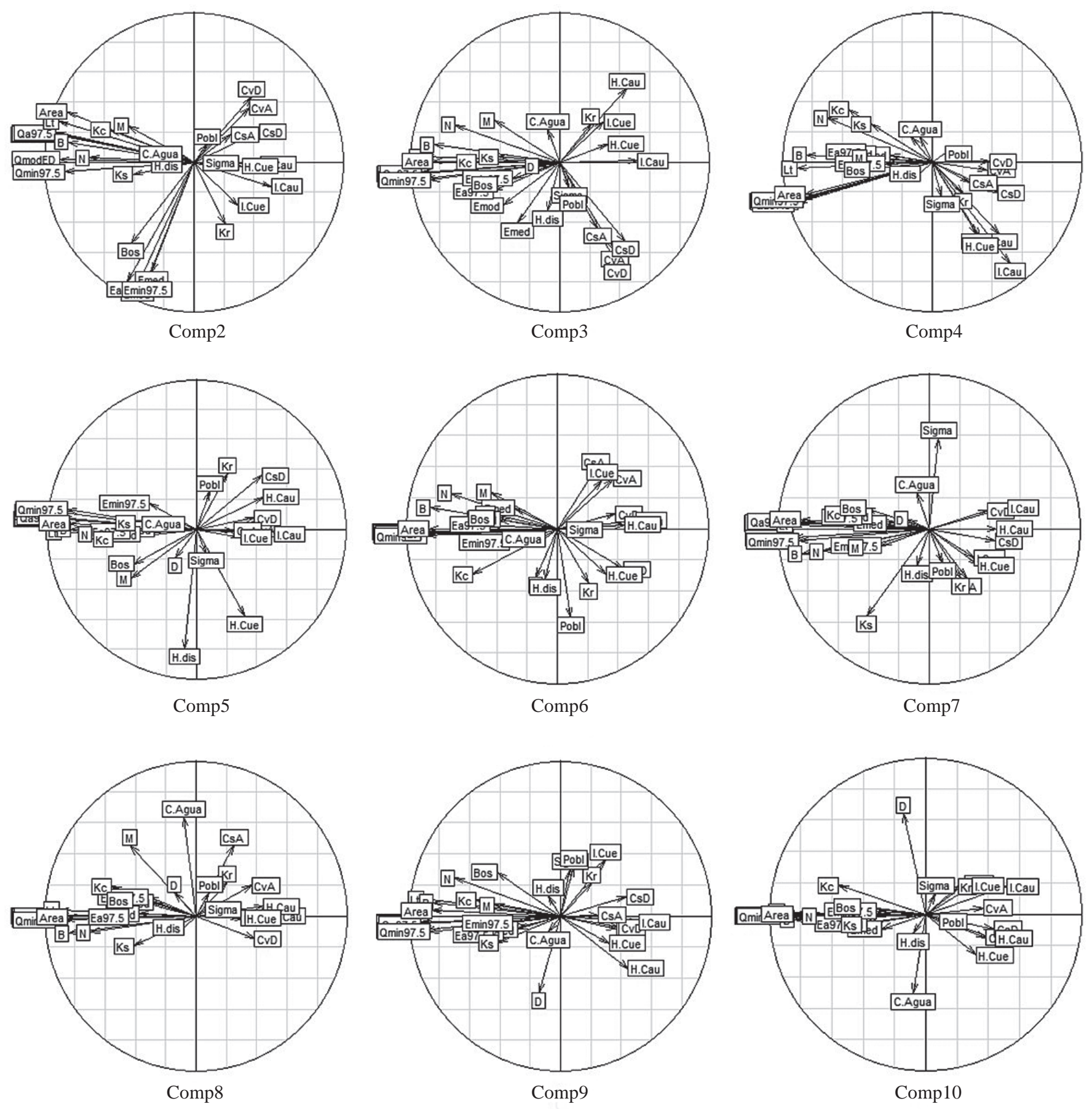

Figura 6. Biplot de las variables en cada Componente para las variables hidrológicas, morfométricas y biofísicas.

hidrológicas, morfométricas y biofísicas descritas para cada una realmente corresponden a una proporción muy cercana de la realidad.

Si bien los puntos de cierre de cada cuenca fueron sujetos a una modificación respecto a la ubicación original de las estaciones dada por las coordenadas encontradas en el Catálogo de la Red Hidrometeorológica y Ambiental Nacional de Estaciones del IDEAM, se obtuvieron muy bajos errores respecto al error en el área de la cuenca
(Figura 7), y la distancia de movimiento de las estaciones hidrológicas. El 41\% (175 de 421) tuvo una distancia inferior de movimiento a $100 \mathrm{~m}$, el 58\% (244 de 421) inferior a 500m, el $72 \%$ (305 de 421) inferior a 1km, y el 91\% (382 de 421) inferior a $5 \mathrm{~km}$ (Figura 7).

Lo anterior permite pensar que gracias a los buenos ajustes de la red hídrica generada y al buen proceso de depuración en la ubicación del punto de cierre para cada cuenca, son en conjunto un indicador de que las 

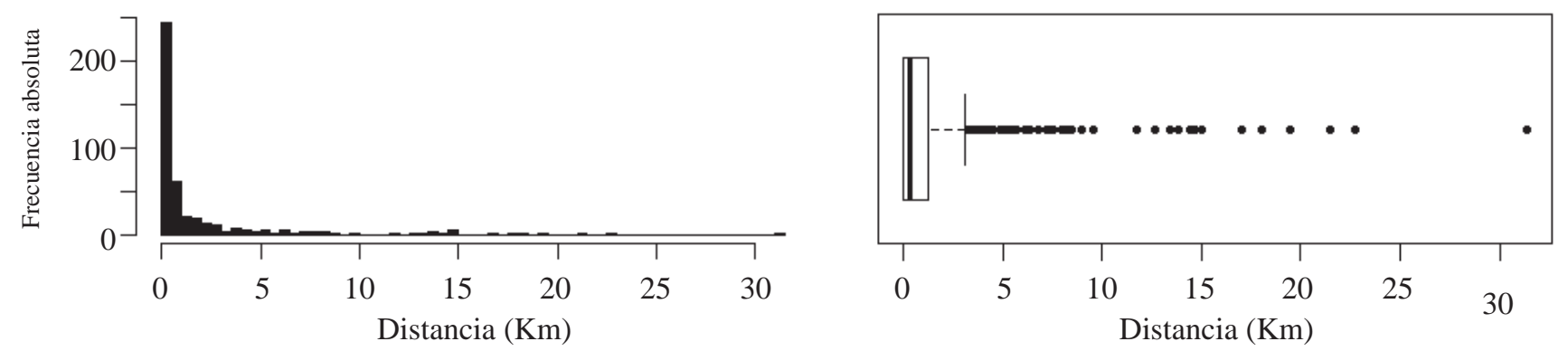

Figura 7. Histograma y Box-Plot para las distancias de movimiento de las estaciones de estudio.

características morfométricas generadas representan con claridad características intrínsecas de cada cuenca (Patton, 1988). Sumado a esto, la significancia encontrada entre las relaciones hidrológicas, morfométricas y biofísicas para la mayoría de las variables, reiteran que el modelo estadístico construido puede contribuir a la estimación de la oferta hídrica en Colombia ante la ausencia y disponibilidad de información.

Analizando las características morfométricas derivadas para cada cuenca se encuentra que en términos generales las cuencas de las regiones de tierras bajas (Amazonia, Orinoquia, Caribe y Pacífico) contrastan respecto a las ubicadas en los Andes. Características como el coeficiente de sinuosidad, el porcentaje de bosques, la pendiente, el ancho de la cuenca, el coeficiente de compacidad, la profundidad y el nivel de disección, son muestra de esto. En estos casos el relieve dominante define la mayoría del comportamiento de estas características (Patton 1988).

Las demás variables morfométricas permanecen poco variantes en el espacio, en principio debido a que las grandes cuencas, que en su mayoría tienen mayores proporciones de área en sus partes bajas contrarrestan los valores superiores que pueden encontrarse en las partes altas. Cabe resaltar características como la profundidad de disección, la cual es bastante mayor en tierras bajas, indica ríos aparentemente superficiales en las zonas altas.

La variable porcentaje de centros poblados la cual se esperaba brindase algunas directrices en términos de conservación del recurso hídrico aparece constante. Se esperaba encontrar cuencas en las que el tamaño de la población inferido a través de la cobertura en centros poblados permitiera definir zonas claves para el estudio de la oferta hídrica en Colombia (Domínguez y Ivanova, 2005; Domínguez, et al., 2008; Domínguez, et al., 2010). En este sentido, si bien la presión del recurso hídrico está definida por la cantidad de personas que acceden a él, no se tienen en cuenta los usos del suelo, ni la proporción de uso de agua de cada uno de éstos en cada cuenca, los cuales pueden ser un factor de mayor asociación con las características hidrológicas, morfométricas y biofísicas de cada cuenca.

Igualmente, la proporción de bosques, aunque es significativamente mayor en las cuencas del Amazonas, Orinoquia, Pacífico y Sierra Nevada de Santa Marta, mientras disminuye en el Centro del País, no presenta una relación clara con los valores de caudal reportados, sí lo hace con los de escorrentía, lo que podría indicar una aparente relación entre la existencia de bosque y la oferta hídrica. No obstante, es conocido que la relación entre la cantidad de agua que escurre por una cuenca está mediada en gran proporción por la precipitación, además de otros factores. Esta relación se comprobó mediante un análisis de regresión lineal usando los residuales de un modelo lineal regresivo escorrentía vs precipitación (tomando el valor promedio de precipitación anual en el área aferente para cada una de las 421 cuencas) y la proporción de bosque. Se encontró que tan solo el 5\% $\left(\mathrm{R}^{2}=\right.$ $0.05, \mathrm{p}<0.01$ ) de las diferencias en los valores de escorrentía entre las cuencas son explicados por las diferencias en su proporción de bosque, mientras la precipitación explica el $75 \%, \mathrm{R}^{2}=0.75 \mathrm{p}<0.01$ (Figura 8). Adicionalmente, otros
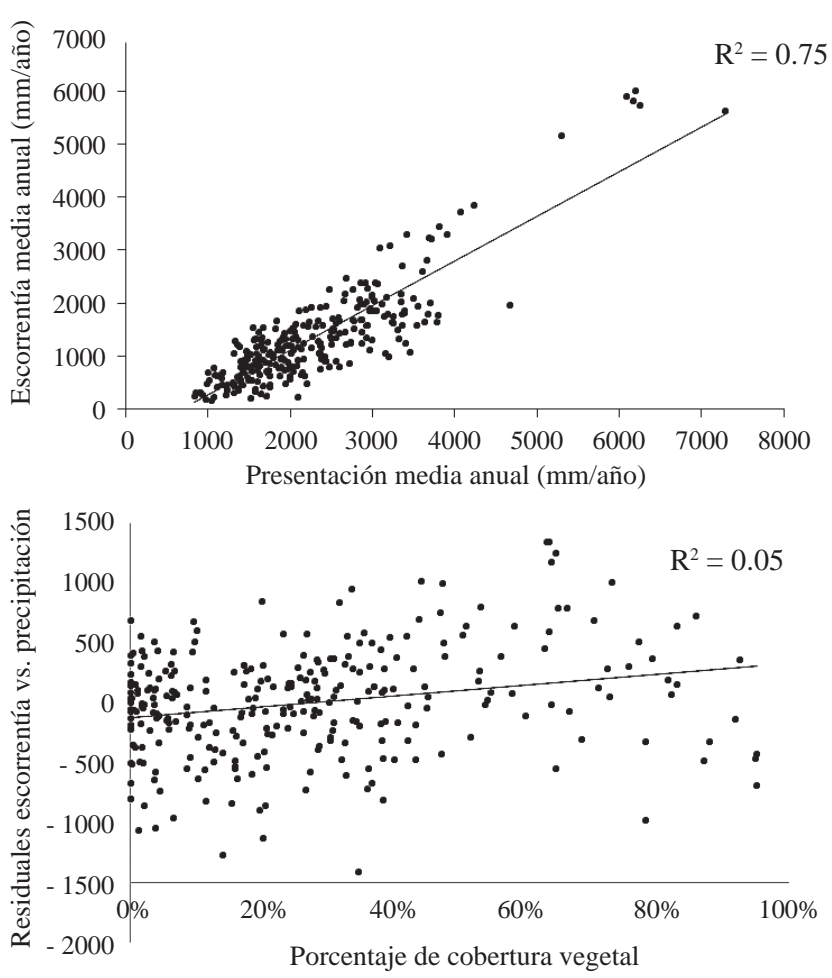

Figura 8. Resultados de la regresión por pasos para determinación de la posible influencia vegetación-escorrentía. 
estudios aplicando modelos determinísticos sugieren que la relación entre la proporción de bosques y el flujo del agua no son perceptibles fácilmente. Distintos reportes usando modelos determinísticos estiman que se necesita al menos una diferencia del $20 \%$ en bosques entre una cuenca y otra para encontrar una relación entre éstos y los flujos simulados (Brunjinzeel, 1990; Johnson, 1998; Wilk, Andersson, Plermkamon, 2001; Burbano-Girón, et al., 2009).

De lo anterior es posible verificar con próximos estudios si existe una relación entre la fragmentación y la oferta hídrica, ya que aparentemente en cuencas donde hay una mayor proporción de bosque hay mayor oferta hídrica, no obstante esta se encuentra en lugares donde la proporción de la demanda por el agua es la menor. En esta medida cabría investigar con herramientas de modelamiento no determinísticas, ya que se ha visto que estas no son sensibles ante cambios en la cobertura (Johnson, 1998; Burbano-Girón, et al., 2009), si ante posibles eventos de reforestación de los Andes donde se sabe la presión por el uso de recursos boscosos e hídricos es mayor (Etter y Van Wyngaarden, 2000; Etter y Villa, 2000; Etter, et al., 2006; Domínguez, et al., 2008; Etter y McAlpine, 2008; Domínguez, et al., 2010), se podría favorecer la oferta hídrica gracias a procesos de conectividad hidrológica (Freeman, Pringle y Jackson, 2007).

Por otra parte cabe resaltar la relación inversa que existe entre los valores de caudal y sus respectivos coeficientes de variación y asimetría, lo que implica aparentemente una relación también inversa con la escorrentía y la proporción boscosa, indicando que es posible que con predominancia de bosque se presente una mayor regularidad en la oferta hídrica, siendo en estos casos claves procesos de reforestación que permitirían en caso de fluctuación en la disponibilidad del recurso hídrico bajo eventos de cambio climático (Domínguez, et al., 2010), una zona de amortiguación para la oferta de agua en Colombia, especialmente en la región de los Andes.

\section{Conclusiones}

En Colombia la aplicación de un modelo integrado del ciclo hidrológico de cualquier tipo presenta dificultades en términos de adquisición de información y delimitación de la escala de trabajo para la inmensa mayoría de cuencas. En esta medida la generación de información relacionable a la hidrológica del país es clave, por lo que este trabajo en su primera fase inicia la construcción de una base de datos de parámetros morfométricos y biofísicos para ser utilizada en la determinación del impacto del uso del paisaje, sobre los parámetros de modelos estocásticos que describan la evolución de características probabilísticas de los elementos del ciclo hidrológico.

La generación de cuencas en base a DEMs brinda un buen ajuste con relación a las generadas con base a cartografía, aunque el ajuste de posición que normalmente debe hacerse de las estaciones hidrológicas puede generar distorsión en los resultados encontrados. Para este estudio el $91 \%$ de las cuencas generadas tuvo un error relativo inferior al $15 \%$ en el área, con respecto a la reportada por el Catálogo de la Red Hidrometeorológica y Ambiental Nacional de Estaciones del IDEAM, lo que permite pensar que las características hidrológicas, morfométricas y biofísicas descritas para cada una realmente describen la cuenca.

Con el modelo estadístico de ACP aplicado pueden evidenciarse relaciones muy cercanas entre el tamaño de la cuenca, el orden del río y las variables de caudal anual y diario. A pesar de que la escorrentía y la proporción de bosque se agrupan, es claro que esta relación está mediada por la precipitación y tan sólo el 5\% de la escorrentía por la presencia de bosques. Las variables referentes al relieve muestran una cercanía muy grande, la pendiente y altura media de la cuenca y el cauce están muy asociadas, por lo que cualquiera de estas puede ser usada como indicador de la topografía de la cuenca.

La aparente relación inversa existente entre los coeficientes de variación y asimetría y la escorrentía y la proporción boscosa, indican que es posible que la predominancia de bosque favorezca una mayor regularidad en la oferta hídrica, siendo en estos casos claves procesos de reforestación que permitirían en caso de fluctuación en la disponibilidad del recurso hídrico bajo eventos de cambio climático, una zona de amortiguación para la oferta de agua en Colombia, especialmente en la región de los Andes.

\section{Información suplementaria}

Tabla 1S. Matriz de Correlación entre las variables a incluir en el modelo estadístico. Debajo de la diagonal los valores para la correlación de Pearson y arriba de la diagonal sus respectivos valores de significancia. Para la parte superior se muestran en negrilla todas las correlaciones significativas al 5\%, y en la parte inferior las correlaciones mayores o iguales a 0.6. Vea la tabla 1S en: http://www.raccefyn.co/index.php/raccefyn/article/ downloadSuppFile/291/1666

\section{Conflicto de intereses}

Los autores declaran no tener conflicto de intereses.

\section{Referencias}

Beasley, D. B., Knisel, W. G., Rice, A. P. (Eds.). 1991. Proceedings of the Creams/Gleams Symposium: September 27-29, 1989 Athens, Georgia. UGA-CPES-Agricultural Engineering Department, University of Georgia, Tifton, Georgia, USA.

Bruijnzeel, L.A. 1990. Hydrology of moist tropical forests and effects of conversion: a state of knowledge review. Faculty of Earth Sciences. Free University, Amsterdam, The Netherlands.

Burbano-Girón, J., Domínguez, E., Etter, A. 2009. Modelación del efecto de escenarios de cobertura sobre la migración de nutrientes (N, P2O5) en la cuenca alta del río Magdalena (Huila, Colombia). Ingeniería y universidad 13: 7. 
Chow, V. T., Maidment, D. R., Mays, L. W. 1994. Hidrología aplicada. McGraw-Hill, Bogotá, Colombia.

Departamento Administrativo Nacional de Estadística - DANE. 2008. Censo General 2005 Nivel Nacional. Departamento Administrativo Nacional de Estadística, Bogotá, Colombia.

Dolgonosov, B. M., Korchagin, K. A. 2007. A nonlinear stochastic model describing the formation of daily and mean monthly water flow in river basins. Water Resources 34: 624-634.

Domínguez, E. 2004. Aplicación de la ecuación de FokkerPlanck-Kolmogorov para el pronóstico de afluencias a embalses hidroeléctricos (caso práctico de la represa de Betania). Meteorología Colombiana 8: 17-26.

Domínguez, E., Hassidoff, A., León, J., Ivanova, Y., Rivera, H. 2009. Maximal, Minimal and Mean Surface Runoff in Colombia: How is it Distributed? Journal of Environmental Hydrology 17.

Domínguez, E., Ivanova, Y. 2005. Un modelo estocástico para la evaluación hidrológica en alta montaña, bajo condiciones de cambio climático (caso de estudio - páramo de Las Hermosas). Pages 17-30. Bogota.

Domínguez, E., Moreno, J., Ivanova, Y. 2010. Water scarcity in a tropical country?-revisiting the Colombian water resources. International Association of Hydrological Sciences 340: 335-342.

Domínguez, E., Rivera, H. G. 2010. A Fokker--Planck-Kolmogorov equation approach for the monthly affluence forecast of Betania hydropower reservoir. Journal of hydroinformatics 12: 486-501.

Domínguez, E., Rivera, H. G., Sarmiento, R. V., Moreno, P. 2008. Relaciones Demanda-Oferta de Agua y el Índice de Escasez de Agua como Herramientas de Evaluación del Recurso Hídrico Colombiano. Rev. Acad. Colomb. Cienc. XXXII.

Downer, C. W., Ogden, F. L. 2004. GSSHA: Model To Simulate Diverse Stream Flow Producing Processes. Journal of Hydrologic Engineering 9: 161-174.

Environmental Systems Resource Institute - ESRI. 2008. ArcGIS. ESRI, Redlans, California, USA. Retrieved from http://www.esri.com/.

Etter, A., Van Wyngaarden, W. 2000. Patterns of landscape transformation in Colombia, with emphasis in the Andean region. AMBIO: A Journal of the Human Environment 29: 432-439.

Etter, A., Villa, L. A. 2000. Andean forests and farming systems in part of the Eastern Cordillera (Colombia). Mountain Research and Development 20: 236-245.

Etter, A., McAlpine, C. 2008. Chapter 5. Modeling Unplanned Land Cover Change across ScalesModeling Unplanned Land Cover Change across Scales. Pages 81-98 in M. J. Hill and R. J. Aspinall, editors. Land Use Change. Science, Policy and Management. CRC Press, Boca Raton, Florida, USA. Retrieved from http://dx.doi. org/10.1201/9781420042979.ch5.

Etter, A., McAlpine, C., Wilson, K., Phinn, S. and Possingham, H. 2006. Regional patterns of agricultural land use and deforestation in Colombia. Agriculture, Ecosystems y Environment 114: 369-386. doi: 10.1016/j.agee.2005.11.013.

Freeman, M. C., Pringle, C. M., Jackson, C. R. 2007. Hydrologic connectivity and the contribution of stream headwaters to ecological integrity at regional scales1. JAWRA Journal of the American Water Resources Association 43: 5-14.
Frolov, A. V. 2006. Dynamic-stochastic modeling of long-term variations in river runoff. Water resources 33: 483-493.

Frolov, A. V., Vyruchalkina, T. Y., Solomonova, I. V. 2003. Dynamic-Stochastic Modeling of Rivers Rising from Lakes. Water Resources 30: 627-631.

Goodrich, D. C., Unkrich, C. L., Smith, R. E., Woolhiser, D. A. 2006. KINEROS2-new features and capabilities. Page Proceedings of the Joint Federal Interagency Conference.

Habets, F., Noilhan, J., Golaz, C., Goutorbe, J. P., Lacarrère, P., Leblois, E., Ledoux, E., Martin, E., Ottlé, C., VidalMadjar, D. 1999a. The ISBA surface scheme in a macroscale hydrological model applied to the Hapex-Mobilhy area: Part I: Model and database. Journal of Hydrology 217: 75-96. doi: doi: DOI: 10.1016/S0022-1694(99)00019-0.

Habets, F., Noilhan, J., Golaz, C., Goutorbe, J. P., Lacarrère, P., Leblois, E., Ledoux, E., Martin, E., Ottlé, C., Vidal-Madjar, D. 1999b. The ISBA surface scheme in a macroscale hydrological model applied to the HapexMobilhy area: Part II: Simulation of streamflows and annual water budget. Journal of Hydrology 217: 97-118. doi: 10.1016/S0022-1694(99)00020-7.

Instituto de Hidrología, Meteorología y Estudios ambientales IDEAM. 2008. Estudio Nacional del Agua. IDEAM.

Instituto Geográfico Agustín Codazzi - IGAC. 2002. Atlas de Colombia. Instituto Geográfico Agustín Codazzi, Bogota.

Jarvis, A., Rubiano, J., Nelson, A., Farrow, A., Mulligan, M. 2004. Practical use of SRTM data in the tropics: comparisons with digital elevation models generated from cartographic data. Centro Internacional de Agricultura Tropical - CIAT. 32.

Johnson, R. 1998. The forest cycle and low river flows: a review of UK and international studies. Forest Ecology and Management 109: 1-7.

Karasev, I. F. 2008. The ecological regime based nomenclature of water discharges in river systems. Russian Meteorology and Hydrology 33: 670-675.

Knisel, W. G. 1980. CREAMS: A field-scale model for non-point source pollution evaluation. Pages 11-13 Proc. Non-point Pollution Control Tools and Techniques for the Future Symposium. Gettysburg, PA. June.

Konovalov, V. G. 2007. Long-term changes of water balance components in the basins of rivers fed by snow and ice. Russian Meteorology and Hydrology 32: 529-537.

Leonard, R. A., Knisel, W. G., Still, D. A. 1987. GLEAMS: Groundwater loading effects of agricultural management systems. Trans. ASAE 30:1403-1428.

Lindström, G. 1997. A simple automatic calibration routine for the HBV model. Nordic hydrology 28: 153-168.

Lørup, J.K., Refsgaard, J.C. y Mazvimavi, D. (1998) Assessing the effect of land use change on catchment runoff by combined use of statistical tests and hydrological modelling: Case studies from Zimbabwe. Journal of Hydrology, 205: 147-163.

Martin, P. H., LeBoeuf, E. J., Dobbins, J. P., Daniel, E. B., Abkowitz, M. D. 2005. Interfacing GIS with Water Resource Models: A State-Of-The-Art Review. JAWRA Journal of the American Water Resources Association. 41: 1471-1487.

Mesa, O., Poveda, G., Carvajal, L. F. 1997. Introducción al Clima de Colombia. Universidad Nacional de Colombia, Medellín, Colombia. 
Noilhan, J., and J.-F. Mahfouf. 1996. The ISBA land surface parameterisation scheme. Global and Planetary Change. 13: 145-159. Doi: doi: DOI: 10.1016/0921-8181(95)00043-7.

Patton, P. C. 1988. Drainage Basin Morphometry and Floods. Pages 51-64 in V. R. Baker, R. C. Kochel, and P. C. Patton, editors. Flood Geomorphology. Wiley-Interscience.

Peña, D. 2002. Análisis de datos multivariantes. McGraw-Hill Interamericana de España, Madrid, España.

Python Software Foundation. 2008. Python 2.6. Python Software Foundation.

R Development Core Team. 2011. R: A language and environment for statistical computing. R Foundation for Statistical Computing, Vienna, Austria. Retrieved from http://www.Rproject.org/.

Samokhin, A., y Saloviov, N. (1980). Hydrology Handbook (First Edition.). Leningrad: Guidrometeoizdat, 296 p.

Singh, V. P., and D. K. Frevert (Eds.). 2002a. Mathematical models of large watershed hydrology. Water Resources Publications, Highlands Ranch, Colorado, USA.

Singh, V. P., and D. K. Frevert (Eds.). 2002b. Mathematical models of small watershed hydrology and applications. Water Resources Publications, Highlands Ranch, Colorado, USA.

Singh, V. P. (Ed.). 1995. Computer models of watershed hydrology. Water Resources Publications, Highlands Ranch, Colorado, USA.

Singh, V. P., y D. K. Frevert. 2006. Chapter 1. Introduction. Páginas 2-19 en V. P. Singh y D. K. Frevert, editores. Watershed Models. CRC Press.
Singh, V. P., y D. K. Frevert (Eds.). 2006. Watershed Models. CRC Press.

USACE - US Army Corps of Engineers, HEC - Hydrologic Engineering Centre. 2009. Geospatial hydrologic modeling extension HEC-GeoHMS. Davis, California, USA.

Validation Team: METI/ERSDAC, NASA/LPDAAC, USGS/ EROS. 2009. ASTER Global DEM Validation, Summary Report. Retrieved from http://www.ersdac.or.jp/GDEM/E/ image/ASTERGDEM_ValidationSummaryReport_Ver1. pdf, last access April 2010.

Viessman, W., and G. L. Lewis. 1996. Introduction to hydrology. HarperCollins College Publishers, New York, USA.

Viney, N., and M. Sivapalan. 2000. LASCAM: The large scale catchment model, User Manual, version 2. Centre for Water Research, University of Western Australia.

Wilk, J., L. Andersson, and V. Plermkamon. 2001. Hydrological impacts of forest conversion to agriculture in a large river basin in northeast Thailand. Hydrological Processes. 15: 2729-2748.

Yang, J., Reichert, P., Abbaspour, K.C. \& Yang, H. (2007) Hydrological modelling of the Chaohe Basin in China: Statistical model formulation and Bayesian inference. Journal of Hydrology. 340: 167-182.

Zammit, C., M. Sivapalan, N. Viney, and M. Bari. 2003. Improvement of physical basis of conceptual model, LASCAM, with explicit inclusion of within catchment heterogeneity of landscape attributes. Pages 921-926. 